\title{
The effect of military boots on front kick dynamics
}

\author{
Michal Vágner ${ }^{1, *}$, Luboš Tomšovský ${ }^{2}$, James J. Tufano ${ }^{3}$, Petr Kubový ${ }^{2}$ \\ Karel Jelen ${ }^{2}$
}

1 Department of Military Physical Education, Faculty of Physical Education and Sport, Charles University, Prague, Czech Republic

2 Department of Anatomy and Biomechanics, Faculty of Physical Education and Sport, Charles University, Prague, Czech Republic

3 Department of Physiology and Biochemistry, Faculty of Physical Education and Sport, Charles University, Prague, Czech Republic

* Corresponding author: vagner@ftvs.cuni.cz

\section{ABSTRACT}

Front kicks are often used in combat activities that involve the use of kicks and punches, including close combat military training. To mimic real-life combative environments where soldiers execute forceful front kicks while wearing military boots, it is logical to wear these boots during close combat training. However, the effect of military footwear on front kick forces remain unknown. Therefore, the aim of this study was to compare the effects of military boots on front kick dynamics. Six male soldiers $(23.3 \pm 1.7 \mathrm{yr}, 74.3 \pm 6.2 \mathrm{~kg}$, $175.5 \pm 4.5 \mathrm{~cm}$ ) performed eight individual front kicks with no military boots, in bare feet $(N B)$ and with military boots $(M B)$. Peak force (N), impulse (N.s) and time to reach peak force (ms), were measured during each kick. Data were analyzed using paired sample t-tests or nonparametric Wilcoxon pair test and Cohen's d.

Results. Neither peak force $(3180 \pm 647 \mathrm{~N})$ nor impulse $(367 \pm 40 \mathrm{~N} . \mathrm{s})$ of MB were different than NB (3157 $\pm 291 \mathrm{~N}$ and $360 \pm 48 \mathrm{~N} . \mathrm{s}$, respectively) $(p=0.85 ; d=0.04$ and $p=0.36 ; d=0.12$, respectively). Peak force was reached in a significantly shorter time during MB (39 $\pm 16 \mathrm{~ms}$ ) compared to NB (56 $\pm 8 \mathrm{~ms}$; $d=-0.88$ ).

Conclusion. Peak force was reached in a shorter time using $M B$ compared to barefoot $(N B)$ front kicks. The use of military boots changes the time course of peak force during the front kick.

\section{KEYWORDS}

close combat; military equipment; kinetics; impact forces

DOI

10.14712/23366052.2018.10

(c) 2018 The Authors. This is an open-access article distributed under the terms of the Creative Commons Attribution License (http://creativecommons.org/licenses/by/4.0), which permits unrestricted use, distribution, and reproduction in any medium, provided the original author and source are credited. 


\section{INTRODUCTION}

Although there are many different types of combat activities, one of the most common is close combat. In contrast to other types of combat, such as martial paths, martial sports, or others that are performed for different purposes (Martínková \& Parry, 2016; Martínková \& Vágner, 2010), close combat is characterized by maximal effort punches, kicks, and throws intended to strike, disrupt, or immobilize another individual (Vágner, 2008). Specifically, when talking about pragmatic techniques within the military, close combat comes to mind.

In combat environments, military personnel wear personal protective equipment $(P P E)$ that typically consists of a helmet and thorax-protection system (i.e. a vest with ceramic plates) (Swain, Onate, Ringleb, Naik, \& Demaio, 2010), military boots, and other tactical gear depending on the soldier's purpose. To mimic real-life combative scenarios, it is logical that close combat should be performed while wearing military equipment and military boots, but the physiological, kinetic, and kinematic effects of wearing such equipment have not been heavily investigated.

Of the multitude of close combat striking techniques, the front kick is one of the most commonplace. The kinetics and kinematics of front kicks have been investigated previously, (Wasik, Czarny, Malolepszy, \& Drozdek-Malolepsza, 2015), and although these studies provide valuable data regarding front kick velocity (Pozo, Bastien, \& Dierick, 2011; Wilk, McNair, \& Feld, 1983) and forces (Dworak, Dziewiecki, \& Maczynski, 2005; Kuragano \& Yokokura, 2012), they have all been conducted in the absence of PPE and military boots. One study has shown that wearing PPE increases the impact force of front kicks (Vágner et al., in press), but the effect of military boots was not investigated. However, the PPE elements used in this study did not contain military boots either. Given that front kick kinetics are influenced by kicking technique (Sorensen, Zacho, Simonsen, Dyhre-Poulsen, \& Klausen, 1996) and the goal of the kick (Ortenburger, Wasik, \& Gora, 2016), it is possible that wearing military boots can result in different front kick dynamics.

Therefore, the aim of this study was to investigate the influence of military boots $(M B)$ on front kick dynamics. Based on previous research (Wilk et al., 1983; Vágner et al., in press; Ortenburger et al., 2016), we hypothesized that front kicks performed in $\mathrm{MB}$ would result in greater impact forces and a quicker time to reach peak force compared to front kicks performed without military boots, in bare feet $(N B)$.

\section{MATERIAL AND METHODS}

This quantitative study was based on an intrapersonal comparison using a quasi-experimental design. Ethical approval was obtained from the Ethics Committee of the Faculty of Physical Education and Sport (No. 025/2016, 5 February 2016). All participants signed the informed consent before the commencement of the experimental testing. All procedures were performed in accordance with the Declaration of Helsinki.

\section{Participants}

Six male soldiers $(22.2 \pm 1.5 \mathrm{y}, 78.8 \pm 5.8 \mathrm{~kg}, 180.6 \pm 4.8 \mathrm{~cm})$ from the Military Department at the Faculty of Physical Education and Sport, Charles University participated 
in the study. The soldiers were able to execute a front kick with proper technique, regularly participating in close combat training. All of the soldiers were healthy for the duration of the experiment and did not suffer from any health problems during measurements.

\section{Experimental protocol}

All measurements were completed in a single laboratory visit, which lasted approximately 40 minutes. After a 10-minute dynamic warm-up consisting of exercises for kicking in combat activities, each participant executed pre-test five front kicks of progressively increasing intensity from $50 \%$ maximum effort with $N B$ and $M B$ to get used to the feeling of kicking against the force plate. The familiarization phase was also used to measure the distance from the force plate for each participant to execute each kick in the same, comfortable position during the testing. These individualized distances were then recorded and used to ensure the same starting position for each kick. All front kicks began with a front posture and were executed so that the foot made contact at a mid-range height, typical of the abdomen or solar plexus (Vágner et al., in press). The order of testing conditions was randomized, and participants executed a single set of eight front kicks with $N B$ or $M B$. Between each kick, participants were given $30 \mathrm{~s}$ of rest, and after the eighth kick of the first condition, 6 min rest was given before repeating the same protocol with the remaining condition.

\section{Measuring device and the gear of participants}

The dynamics of each front kick was measured using a triaxial force plate Kistler 9281 (Kistler, Winterthur, Switzerland) measuring at $1000 \mathrm{~Hz}$ (Svoboda, Soukup, Jelen, \& Kubový, 2016). In the familiarization phase, the lower limit of the magnitude of acting force was set to $100 \mathrm{~N}$ to reduce noise on the force plate and to mark the beginning and the end of the interaction between a participant's foot and the force plate (Vágner et al., in press). The force plate was adjustable along the vertical axis to ensure that the height of the plate was individualized to each participant's "mid-range" height (Dworak et al., 2005; Kuragano et al., 2012). A kick pad (Figure 1) was used to cover the metal surface of the force plate to minimize any potential risk of injury. Using a unique impact device of own design, it was empirically verified that the kick pad absorbed some of the energy of the kick and it reduced the transmission of force. The device allowed us to analyze the influence of various impact velocities and a different size of contact surface on the impact force measurements. Supposing that the impact velocity of a foot does not exceed $10 \mathrm{~m} / \mathrm{s}$ during a front kick, the absorption coefficients of the kick pad for the impulse and the peak force were found constant up to this value. Specifically, the absorption coefficients were 0.844 for the impulse and 0.167 for the peak impact force. It was also shown that there was no significant influence of a contact surface size ranging by $\pm 30 \%$ on the absorption coefficients. The absorption coefficients were used to determine absolute values of physical quantities measured in this research. The absolute values can be compared to the results of other similar studies.

All participants wore standardized military field clothing and boots designed for the Armed Forces of the Czech Republic. The standardized military boots (model 2000) had a hydrophobic cow leather upper and a SLAVEX rubber sole. 


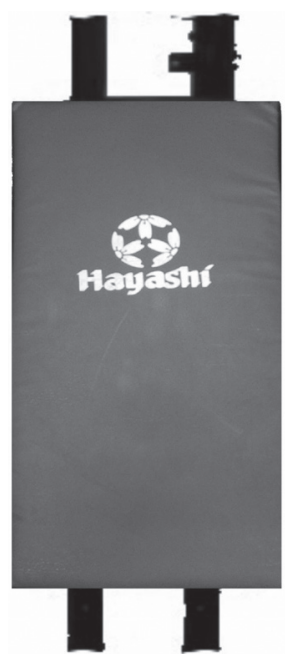

Figure 1 Force plate with kick pad (Hayashi, Size: $75 \times 35 \times 15 \mathrm{~cm}$ )

\section{Measurement procedure and data collection}

Several physical quantities describing the dynamics of a front kick were determined for both conditions (Vágner et al., in press). Peak force $\left(\overrightarrow{F_{\text {peak }}}\right)$ was determined as the sum of force exerted in all three directions $\mathrm{x}, \mathrm{y}, \mathrm{z}$ (Dworak et al., 2005) (Eq. 1).

$\left|\overrightarrow{F_{\text {peak }}}\right|=\max \left(\sqrt{\overrightarrow{F_{x}^{2}}+\overrightarrow{F_{y}^{2}}+\overrightarrow{F_{z}^{2}}}\right)$

The time to reach peak force $(t)$ was defined as the time period between the initial contact of participant's foot with the force plate $\left(t_{0}\right)$ and the peak force reached $\left(t_{\text {peak }}\right)$ (Eq. 2).

$t=t_{\text {peak }}-t_{0}$

The impact force acting during the whole time $\left(t_{\max }\right)$ of a front kick was derived from the formula for the impulse (Eq. 3).

$\vec{I}=\int_{t_{0}}^{t} \vec{F} d t$

Equation 3 was modified for the discrete data measured in this study. For each time pe$\operatorname{riod}(\Delta t)$, defined by the sampling frequency, the impulse was calculated. By summing them together, the overall net impulse of a whole front kick was determined (Eq. 4). The impact force for a whole kick was then derived.

$\overrightarrow{I_{n e t}}=\sum_{i=1}^{n}(\vec{F})_{i} \cdot \Delta t_{i}$ 
An example of a force-time curve for one front kick without military boots is shown in Figure 2. Time to reach peak force was defined as the time difference between the initial foot contact $\left(t_{0}\right)$ and the peak force $\left(F_{\text {peak }}\right)$. For impulse analysis, we used the course of the whole kick.

An example of a force-time curve for one front kick with $M B$ is shown in Figure 3.

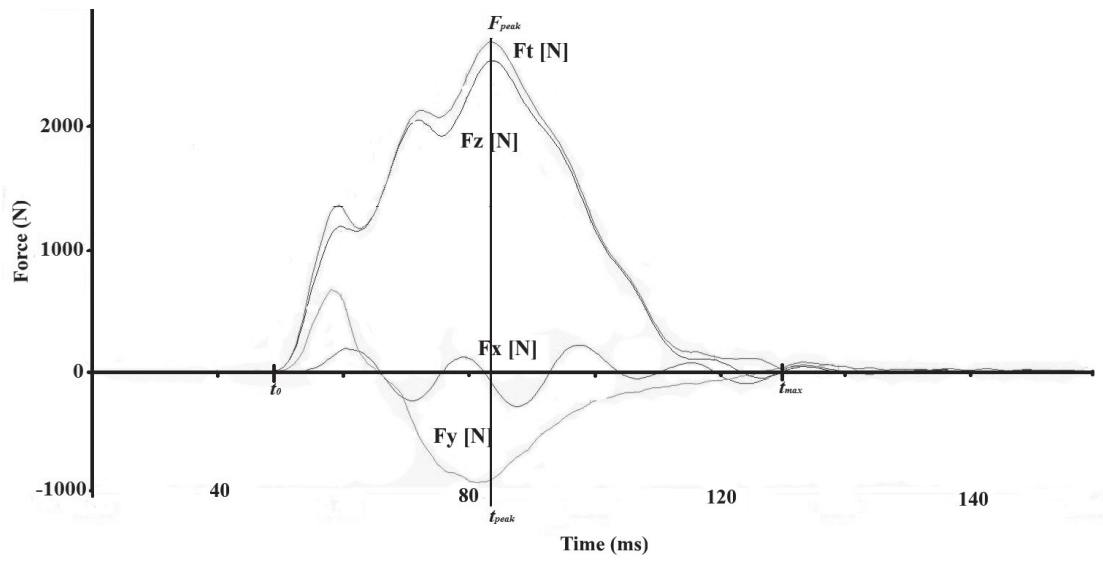

Figure 2 The time course of the kinetic force of one front kick with NB

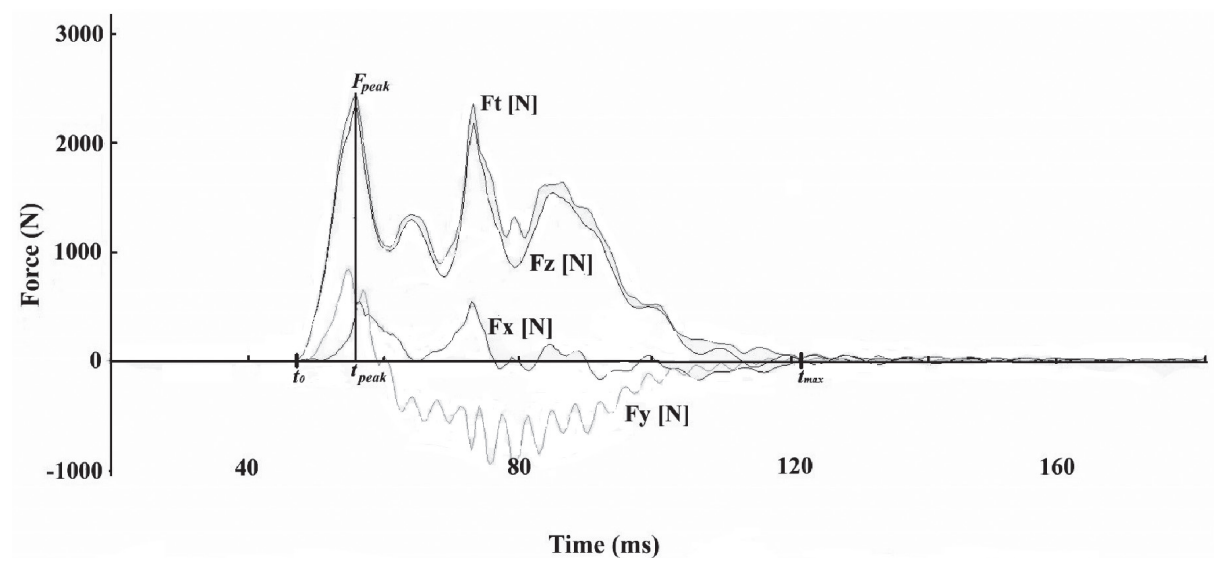

Figure 3 The force-time curve of the impact force for one front kick with MB

\section{Statistical Analysis}

The mean and standard deviation of all eight kicks were calculated for each condition ( $N B$ and $M B$ ). The Shapiro-Wilk test was used to determine whether the data were normally distributed. The non-parametric Wilcoxon paired test was chosen for data that were not of a normal distribution. In case of a normal distribution, the parametric 
paired sample t-test was used. The significance level alpha $=0.01$ was chosen for all statistical analyses. Cohen's D was used to determine the effect size.

\section{RESULTS}

There was neither a significant difference for peak force $(p=0.85 ; d=0.04)$ (Figure 4 ) nor impulse ( $p=0.36 ; d=0.12$ ) (Figure 5 ) between conditions.

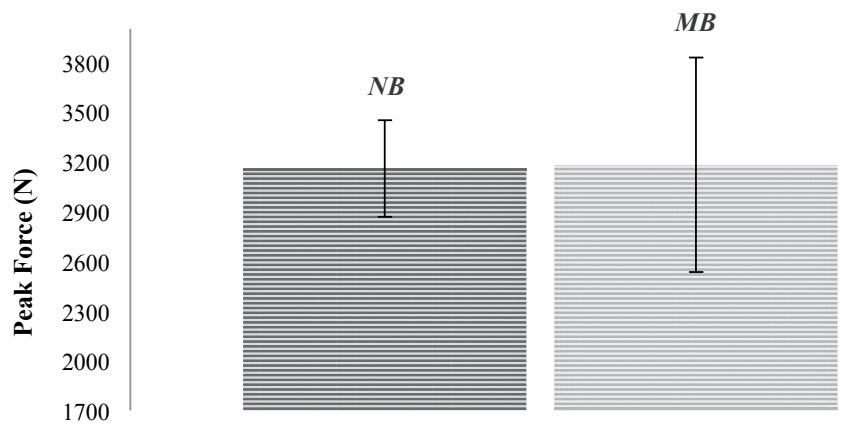

Kicks

Figure 4 Peak force with military boots (MB), and no boots (NB)

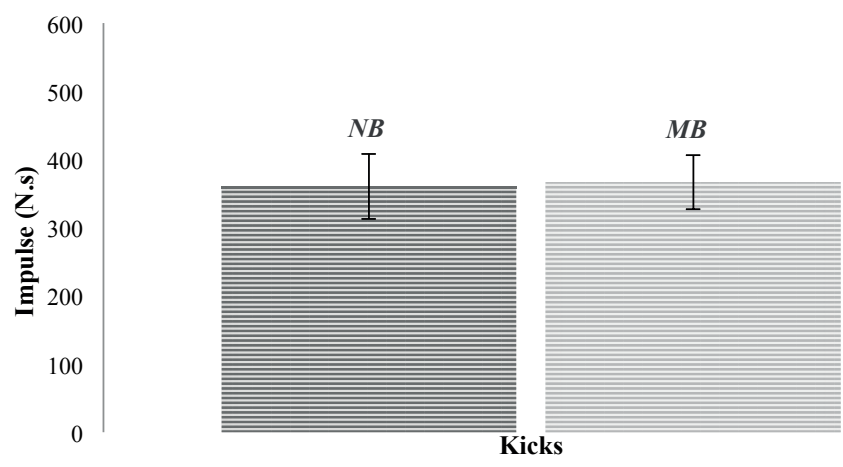

Figure 5 Results of impulse with military boots (MB) and no military boots (NB)

The time to reach peak force was significantly less during $M B$ compared to $N B$ $(d=-0.88)$ (Figure 6).

\section{DISCUSSION}

The results of this study showed no significant difference for the peak force or the impulse between conditions. However, it was demonstrated that front kicks with military boots significantly reduced the time needed to reach peak force.

The peak force and impulse of front kicks with $M B(3180 \pm 647 \mathrm{~N}$, respectively $367 \pm 40$ N.s $)$ was not different than $N B(3157 \pm 291 \mathrm{~N}$, respectively $360 \pm 48$ N.s $)$. 

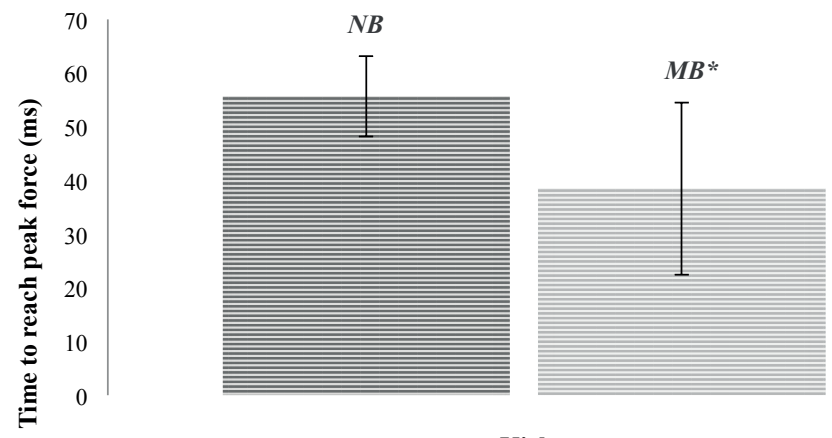

Kicks

${ }^{*}$ significantly less than $N B(p<0.01)$

Figure 6 Time to reach peak force with military boots (MB), and no boots (NB)

However, we found something interesting, as peak force was achieved quicker in $M B$ ( $39 \pm 16 \mathrm{~ms}$ ) compared to $N B(56 \pm 8 \mathrm{~ms})$. In another study, Vágner et al. (in press) found peak force with $M B$ was $5201 \mathrm{~N}$, impact force $3405 \mathrm{~N}$ and time to reach peak force $14.02 \pm 6.71 \mathrm{~ms}$. In this study participants kicked into the tatami $(8 \mathrm{~cm})$, which it was fixed on the force plate. In our study, a kick pad was fixed on the force plate. The kick pad was wider and softer. This also causes that the time to reach peak force is longer than the study of Vágner et al. (in press). The results of our study are hard to compare to other studies that focused mainly on barefoot front kicks. Nevertheless, Kurgano \& Yokokura (2012) found that the peak force of font kicks performed by Japanese martial art Nishon-Kempo was 4500 N. Dworak et al. (2005) found that the impact force was $2900 \mathrm{~N}$.

Interesting findings have also occurred when viewing the kick progress with $M B$ (Figure 3). During the kick, we measured two peak forces that were almost the same. Probably, this is caused by the interaction of the military boots with contact of the kick pad on the force plate, where the military boots hit and pressed the kick pad. There was no such thing as a kick with $N B$ (Figure 2).

The intrapersonal comparison of the peak force showed no significant differences for four participants between using military boots or not during front kicks. More-detailed analysis of the results for other two participants came to contradictory conclusions. The peak force was higher when using no boots compared to using military boots for one participants and in case of the other one, the results were opposite.

Small sample size and the analysis of only dynamics of front kicks without any kinematical analysis are the limitations of the study. Future research should focus on the analysis of both, dynamics and kinematics of front kicks. However, this is the first study analyzing the effect of military boots on the dynamics of front kicks in trained soldiers.

\section{CONCLUSIONS}

Wearing military boots decreased the time to reach peak force during the front kick. There was a different shape of the force-time curve between both conditions during 
front kicks into the kick pad fixed on the surface of the force plate. Using military boots resulted in two peaks in the force-time curve, of which the second one reached a lower maximum. Military boots changed the course of impact force a front kick.

\section{ACKNOWLEDGEMENTS}

Close combat - physical confrontation between two or more opponents at short range involving weapons (knife, stick, firearms and other distance weapons).

Personal protective equipment - protective clothing, helmets, thorax-protection system or other equipment designed to reduce the likelihood of serious injury from the impact of small arms fire and fragments.

Front kick - is a kick executed by lifting the knee straight forward, while pulling the foot to the hamstring, and then straightening the leg in front of the target area.

Impact force - the force generated at the start of contact or collision. In close combat, this is the impact of a kick or punch as they hit the body or solid pad.

The research was supported by grant SVV 260466.

\section{REFERENCES}

Dworak, L. B., Dziewiecki, K., \& Maczynski, J. (2008). Characteristics of kinematics and kinetics of strokes in karate-biomechanical approach. In: ISBS-Conference Proceedings Archive, 1(1).

Kuragano, T., \& Yokokura, S. (2012). Experimental analysis of Japanese martial art Nihon-Kempo. The ICHPER-SD Journal of Research in Health, Physical Education, Recreation, Sport \& Dance, $7(1), 40$.

Martínková, I., \& Parry, J. (2016). Martial categories: clarification and classification. Journal of the Philosophy of Sport, 43(1), 143-162.

Martínková, I., \& Vágner, M. (2010). Terminologické vymezení bojových aktivit v oblasti kinantropologie. Česká kinantropologie, 14(1), 29-38.

Ortenburger, D., Wasik, J., \& Gora, T. (2016). Selected dimensions of the self-esteem and a kinematic effect of the intentional target at taekwondo athletes. Archives of Budo Science of Martial Arts and Extreme Sports, 12, 117-121.

Pozo, J., Bastien, G., \& Dierick, F. (2011). Execution time, kinetics, and kinematics of the mae-geri kick: Comparison of national and international standard karate athletes. Journal of sports sciences, 29(14), 1553-1561.

Sorensen, H., Zacho, M., Simonsen, E. B., Dyhre-Poulsen, P., \& Klausen, K. (1996). Dynamics of the martial arts high front kick. Journal of sports sciences, 14(6), 483-495.

Swain, D. P., Onate, J. A., Ringleb, S. I., Naik, D. N., \& DeMaio, M. (2010). Effects of training on physical performance wearing personal protective equipment. Military medicine, 175(9), 664-670.

Vágner, M. (2008). K teorii boje zblizka. Prague: Karolinum.

Vágner, M., Thiel, D., Jelen, K., Tomšovský, P., Kubový, P., Moska, W., Lulińska-Kuklik, E., Cięszczyk, P., Maszczyk, A., \& Tufano J. J. Wearing ballistic and weighted vests increases front kick forces. Archives of Budo [In press].

Wasik, J., Czarny, W., Malolepszy, E., \& Drozdek-Malolepsza, T. (2015). Kinematics of taekwon-do front kick. Archives of Budo Science of Martial Arts and Extreme Sports, 11, 23-28.

Wilk, S. R., McNair, R. E., \& Feld, M. S. (1983). The physics of karate. American Journal of Physics, 51(9), 783-790. 